\title{
Hedgehog signaling is non-cell autonomously activated in the cystic kidney of $\mathrm{Arl13b}$ mutant mice
}

Chia-Ling Hsieh, Stephanie Justine Jerman, Zhaoxia Sun*

Department of Genetics, Yale University School of Medicine, 333 Cedar St. New Haven, CT 06520, USA

*: Corresponding author

email: zhaoxia.sun@yale.edu

Running title: Non-cell autonomous Hedgehog activation in a PKD mouse model

Key words: Arl13b, Cilia, epithelial-interstitial communication, Hedgehog signaling, polycystic kidney disease (PKD) 


\section{ABSTRACT:}

Background: Polycystic kidney disease (PKD) is a ciliopathy characterized by fluid-filled epithelial cysts in the kidney. Although it is well established that the primary cilium is essential for Hedgehog $(\mathrm{HH})$ signalling and $\mathrm{HH}$ signalling is abnormally activated in cystic kidneys of multiple PKD models, the mechanism and function of $\mathrm{HH}$ activation in PKD pathogenesis remains incompletely understood.

Methods: We used a transgenic $\mathrm{HH}$ reporter line to identify the target tissue of $\mathrm{HH}$ signalling in $A r l 13^{f f f} ; K s p$-Cre mutant kidney, in which the cilia biogenesis gene Arll3b is specifically deleted in epithelial cells of the distal nephron. In addition, we treated Arl13b $b^{f / f}$;Ksp-Cre mice with the GLI inhibitor GANT61 and analyzed its impact on PKD progression in this model.

Results: In vivo in the mouse kidney, deletion of Arll3b in epithelial cells led to non-cell autonomous activation of the $\mathrm{HH}$ pathway in the interstitium. In addition, whole body inhibition of the HH pathway by GANT61 reduced cyst burden, suppressed fibrosis and reduced kidney function decline in $\mathrm{Arll} 3 \mathrm{~b}^{f / f} ; \mathrm{Ksp}$-Cre mice. 
Conclusions: Our results reveal non-cell autonomous activation of $\mathrm{HH}$ signalling in the interstitium of the cystic kidney of $\mathrm{Arll} 3 \mathrm{~b}^{f f} ; \mathrm{Ksp}$-Cre mice and suggest that abnormal activation of the $\mathrm{HH}$ pathway contributes to $\mathrm{PKD}$ progression.

\section{INTRODUCTION:}

The primary cilium is a microtubule-based cellular organelle that protrudes from the cell surface into the extracellular matrix or fluid, and functions as an antenna to sense and transduce environmental signals to regulate cellular responses ${ }^{1-3}$. One of the best studied cilia-regulated pathways is the Hedgehog $(\mathrm{HH})$ pathway. Three secreted ligands, Sonic hedgehog (SHH), Indian hedgehog (IHH) and Desert hedgehog (DHH), initiate HH signaling by binding to the receptor PATCHED1/2 (PTCH1/2). In vertebrates, PATCHED is localized to cilia in the absence of its ligands. Binding of ligands activates the $\mathrm{HH}$ pathway by displacing ciliary PTCH and allows the G-protein coupled receptor-like protein SMOOTHENED (SMO) to enter the cilium. SMO accumulation facilitates processing of

GLI proteins, which are then trafficked to the nucleus and transcribe $\mathrm{HH}$ target genes, including Gli1, Ptch1 and Ptch2 ${ }^{4-6}$. A functional cilium is therefore required for the 
activation of the $\mathrm{HH}$ pathway. The $\mathrm{HH}$ signaling pathway plays a fundamental role in tissue patterning, cell growth and differentiation during development ${ }^{7-9}$. HH signaling is frequently reactivated during injury and repair in multiple organs, including the lung and kidney, and prolonged $\mathrm{HH}$ activation contributes to tissue fibrosis ${ }^{10-14}$.

Mutations in ciliary genes, encoding proteins required for cilia formation or function, contribute to a wide spectrum of human disorders, including polydactyly, holoprosencephaly, obesity and polycystic kidney disease (PKD), among many others, collectively known as ciliopathies ${ }^{15-17}$. While abnormal HH signaling underlies multiple ciliopathies such as polydactyly and craniofacial ciliopathies, the molecular mechanism of PKD pathogenesis remains poorly understood ${ }^{18,19}$. Autosomal dominant polycystic kidney disease (ADPKD), the most frequent form of PKD and one of the most common monogenetic diseases in human, can be attributed to mutations in PKD1 and PKD2, which encode POLYCYSTIN 1 and 2 (PC1 and PC2), respectively (reviewed in ${ }^{20}$ ). PC1 and PC2 are trafficked to cilia and their ciliary localization is integral to their function ${ }^{21-25}$. It is postulated that PC1 and PC2 functions to inhibit a cilia-dependent and cyst promoting 
pathway (CDCA) and unconstrained CDCA leads to cyst formation in Pkd1 or Pkd2 mutants ${ }^{26}$. However, the precise molecular mechanism of this pathway is undefined.

Abnormal activation of the HH pathway has been detected in cystic kidney samples from both human and animal models, including Thm1 mouse mutants ${ }^{27-30}$. Thm1/Ift139 encodes a component of the IFT A complex that negatively regulates $\mathrm{HH}$ signaling ${ }^{31}$. Defective Thm1 leads to shorter cilia with bulbous tips, upregulation of HH signaling and cystic kidney ${ }^{27,31}$. In concordance with Thm l's inhibitory role in HH signaling, repressing the $\mathrm{HH}$ pathway reduced cyst progression in the Thm1 mutant kidney ${ }^{27}$. Moreover, cyst expansion induced by $c A M P$ and $P k d l$ inactivation in cultured mouse kidney explants was also shown to be sensitive to $\mathrm{HH}$ inhibition ${ }^{27}$. In the meantime, a recent study demonstrated that modulating the $\mathrm{HH}$ pathway in renal epithelial cells failed to affect PKD progression in mouse $P k d 1$ mutants $^{32}$, suggesting that the role of $\mathrm{HH}$ signaling in different PKD models is complex and not fully understood.

In a previous study, we found that the $\mathrm{HH}$ pathway is upregulated in the cystic kidney of $A r l 13 b^{f f} ; K s p$-Cre mice, where $A r l 13 b$ is specifically deleted in epithelial cells of the distal nephron ${ }^{29}$. Since $A r l 13 b$ is required for cilia biogenesis, this result seems contradictory to the essential role of cilia in $\mathrm{HH}$ signaling. However, $\mathrm{HH}$ ligands commonly activate $\mathrm{HH}$ signaling non-cell autonomously during tissue patterning ${ }^{8,9}$. Similarly, following acute kidney injury, SHH is produced by epithelial cells, but downstream targets are upregulated in interstitial cells ${ }^{10,12,33}$. The role of non-cell autonomous HH signaling has not been investigated in PKD pathogenesis and is the focus of this study. 
We demonstrate that $\mathrm{HH}$ signaling is predominantly activated in the interstitium when Arl13b is deleted in renal epithelial cells. In addition, treatment of the GLI inhibitor GANT61 partially suppressed both renal cyst expansion and fibrosis, and preserved kidney function in $\mathrm{Arl13} \mathrm{b}^{f / f} ; \mathrm{Ksp}$-Cre mice. Together, our results clarify the role of renal epithelial and interstitial cells in the abnormal activation of $\mathrm{HH}$ signaling in cystic kidneys and highlight the significance of epithelial-interstitial cross-talk in this process. Our results also suggest that non-cell autonomous HH signaling contributes to both renal cyst progression and fibrosis in $A r l 13 b$ mutant mice, and may provide important insight into the molecular etiology of cystic diseases in human.

\section{RESULTS:}

\section{Hedgehog signaling is activated non-cell autonomously in the interstitium of the} $A r l 13 b^{f / f} ; K s p-C r e$ kidney

We previously showed that the expression of $\mathrm{HH}$ ligands and target genes are upregulated in the cystic kidney of $A r l 13 b^{f / f} ; K s p$-Cre mice in whole kidney lysates ${ }^{29}$. To ascertain tissue specificity of $\mathrm{HH}$ activation in the mutant kidney, we took advantage of the $\mathrm{Gli}^{\mathrm{LacZ} /+}$ reporter line that expresses nuclear localized LacZ in cells with activated $\mathrm{HH}$ signaling ${ }^{34}$. We generated Arl13bff/Ksp-Cre; Gli $1^{\text {LacZ/+ }}$ mice and performed immunofluorescence analysis for LacZ in sections of postnatal day 21 (P21) kidneys. In kidneys with functional ARL13B $\left(\right.$ Arl $\left.13 b^{f /+} ; K s p-C r e ; G l i 1^{\text {LacZ/++}}\right)$, we detected LacZ positive nuclei sporadically in both the cortex and medullar region, outside of epithelial 
tubules, as outlined by anti-Laminin signal, consistent with the known distribution of $\mathrm{Glil}^{+}$ cells in the wild type kidney (Fig. 1A) ${ }^{10}$. In the Arl13bfff;Ksp-Cre; Gli ${ }^{\text {LacZ/+ }}$ mutant kidney, the number of LacZ positive nuclei increased; notably, they were still excluded from regions encircled by Laminin, suggesting that stromal cells were the $\mathrm{HH}$ responding cells (Fig. 1A). Since the morphology of tubules, epithelial cells and the pattern of Laminin signal were significantly distorted in the mutant kidney, to validate the identity of nuclear LacZ (nLacZ) positive cells, we additionally performed co-labelling with anti-LacZ and anti-alpha Smooth Muscle Actin ( $\alpha$ SMA), a marker of activated myofibroblasts. In both the cortex and medullar region of the mutant kidney, the signal of $\alpha \mathrm{SMA}$ increased dramatically, as expected ${ }^{29}$ (Fig. 1B). Moreover, most nLacZ positive cells were also positive for $\alpha \mathrm{SMA}$, suggesting that they were myofibroblasts (Fig. 1B).

Combined, these results suggest that inactivation of $A r l 13 b$ in renal epithelial cells activates $\mathrm{HH}$ signaling in stromal cells via a non-cell autonomous mechanism.

\section{Mesenchymal 10T1/2 and epithelial mIMCD3 cells show differential responsiveness}

\section{to HH stimulation}

Since epithelial cells in the $\mathrm{Arll} 3 \mathrm{~b}^{\mathrm{f}+\mathrm{H}} ; \mathrm{Ksp}-\mathrm{Cre} ; \mathrm{Gli1}^{\mathrm{LacZ} /+}$ kidney are negative for $\mathrm{HH}$ signaling, as indicated by the lack of nLacZ signal (Fig. 1A), we investigated whether mesenchymal and epithelial cells show differential response to $\mathrm{HH}$ stimulation. We cultured mouse inner medullary collecting duct (mIMCD3) cells and the murine mesenchymal cell line 10T1/2 in vitro. After reaching confluency, both cell lines were 
switched to a low serum medium $(0.5 \%$ fetal bovine serum $(\mathrm{FBS}))$ to induce the formation of primary cilia. As expected, abundant cilia could be detected in mIMCD3 cells (Fig. 1C). In addition, 10T1/2 cells also displayed cilia, shown by cilia markers anti-acetylated tubulin and anti-ARL13B (Fig. 1C). Ciliated cells were subsequently treated with SAG, a small molecule agonist of SMO, for 24 hours. The expression level of $\mathrm{HH}$ target genes, including Gli1, Ptch1 and Ptch2, was then analyzed by quantitative RT-PCR (qRT-PCR) to monitor the status of $\mathrm{HH}$ signaling. Even at a low SAG concentration (10nM), expression levels of HH target genes Glil (>143-fold), Ptch1 ( 7-fold) and Ptch2 (12-fold) were significantly increased in stromal $10 \mathrm{~T} 1 / 2$ cells compared to vehicle treated controls (Fig. 1D). In contrast, and despite that mIMCD3 epithelial cells displayed more prominent cilia (Fig. 1C), mIMCD3 cells showed minimal response to SAG, with a slightly increased expression of Gli1 (1.4-fold) at a much higher SAG concentration (500 nM), Ptch1 (1.5 fold at $10 \mathrm{nM} \mathrm{SAG}$ ) and Ptch2 (1.3 fold at $100 \mathrm{nM} \mathrm{SAG}$ ) (Fig. 1E). The limited sensitivity of mIMCD3 cells to activation of $\mathrm{HH}$ signaling is consistent with the lack of $\mathrm{nLacZ}$ signal in renal epithelial cells (Fig. 1A).

\section{The GLI inhibitor GANT61 partially suppresses the renal phenotypes of Arl13b ${ }^{f / f} ;$ Ksp-Cre mice}

Since $\mathrm{HH}$ signaling is activated non-cell autonomously in the Arll $3 b^{f f} ; \mathrm{Ksp}$-Cre kidney, to investigate the functional significance of this abnormal signaling in PKD progression, we used GANT61, a small molecule that interferes with the transcriptional 
activities of both GLI1 and GLI2 ${ }^{35}$, to achieve whole body HH inhibition. Our previous study established that the Arl13b/f;Ksp-Cre kidney is non-cystic at P7 and significantly cystic at $\mathrm{P} 14^{29}$. Because of technical difficulties of intraperitoneal (i.p.) injection into P7 mice, we analyzed key characteristics of the $A r l 13 b^{f f} ; K s p$-Cre kidney at P10 and results showed that the Arl13b/f;Ksp-Cre kidney was slightly cystic at this stage (Fig. 2). The kidney weight to body weight (KWB) ratio, cystic index and blood urea nitrogen (BUN) level were increased as well in both female and male mutant mice (Fig. 2B-G), although at levels much milder than P14 mutants ${ }^{29}$. We therefore started GANT61 treatment from P10. Arll3b $b^{f f} ; K s p-C r e$ and control mice $\left(A r l 13 b^{f /+} ; K s p-C r e\right)$ were subjected to daily i.p. injection of GANT61 or vehicle alone(Figure 3A). After GANT61 treatment, qRT-PCR using total kidney lysates verified that this treatment led to reduced expression level of the HH target genes Gli1, Ptch1 and Ptch2 in mutant kidneys, and to a lesser degree in control kidneys (Fig. 3B). Both female and male mutant mice treated with GANT61 showed significantly reduced kidney weight to body weight (KBW) ratio, while no difference was seen in control animals (Fig. 3C, D). Taking advantage of variations in phenotypic severity between individual mice, we asked whether the level of Glil expression correlates with KBW ratio by Pearson correlation analysis. Interestingly, while there was no significant correlation between Gli1 level and KBW ratio in vehicle treated mutants, the correlation coefficient was statistically significant in GANT61 treated mutants (Fig. 3E, F). Cyst progression, as indicated by cyst index, was also inhibited by this treatment in both male and female mice (Fig. 3G, H, I). By contrast, the same GANT61 treatment had minimal 
effect on kidney size, KBW ratio and cyst formation in control mice, even though the $\mathrm{HH}$ pathway was modestly inhibited in the kidney (Fig. 3B, C, D, H, I).

We then investigated the impact of GANT61 treatment on myofibroblast activation in the kidney of $\mathrm{Arl13} b^{f / f}$;Ksp-Cre mice. Immunofluorescence analysis of kidney sections revealed that VIM and $\alpha$ SMA signals in the interstitium of the renal cortex and medullar regions were significantly decreased in GANT61 treated mutants (Fig. 4A). We further analyzed collagen deposition by trichrome staining. Results revealed that the blue staining, i.e. collagen deposition, was reduced in treated mutants (Fig. 4B). Moreover, Western blot using whole kidney lysates from mutant mice revealed a notable reduction of the protein level of $\alpha$-SMA and collagen I by GANT61 treatment (Fig. 4C).

In concordance with morphologic improvement, the BUN level was significantly reduced in GANT61 treated mutants, indicating preserved kidney function (Fig. 4D, E).

Combined, these results show that the GLI inhibitor GANT61 partially suppresses both cyst progression and fibrosis in the $\mathrm{Arll} 3 \mathrm{~b}^{f f} ; \mathrm{Ksp}$-Cre kidney.

\section{DISCUSSION:}

The primary cilium plays a key role in both PKD pathogenesis and $\mathrm{HH}$ signaling. However, the relationship between the two is less clear and results from different studies are contradictory. For example: It was shown that inhibiting $\mathrm{HH}$ signaling in mouse mutants of Thm1, a negative regulator of $\mathrm{HH}$ signaling, partially suppresses cyst progression $^{27}$. However, in mouse $P k d 1$ mutant models, cell autonomous inhibition of $\mathrm{HH}$ 
signaling, through genetic inactivation of Smo in epithelial cells, does not alter PKD progression $^{32}$. These interesting studies suggest that different PKD or ciliary genes might have distinct relationships with $\mathrm{HH}$ signaling, hence the different response to $\mathrm{HH}$ inhibition. Alternatively, HH signaling could be activated non-cell autonomously in cystic kidneys, resulting in the lack of response by cell autonomous inhibition of $\mathrm{HH}$. We, therefore, tested the mode of $\mathrm{HH}$ activity in a model of cilia dysfunction and $\mathrm{PKD}$, using a $\mathrm{HH}$ reporter in $A r l 13 b^{f f} ; K s p-C r e$ mutant mice. We show that when $A r l 13 b$ is deleted in epithelial cells, HH signaling is activated non-cell autonomously in interstitial cells, consistent with the pattern of $\mathrm{HH}$ activation during development and tissue repair $8-10,12,33$. Non-cell autonomous $\mathrm{HH}$ signaling could be activated through secreted ligands or through mechanical stress exerted by expanding cysts and activate myofibroblast in the stroma (Fig. 4F).

The severe phenotypic consequences of defective epithelial cilia in the $A r l 13 b^{f / f} ; K s p$ Cre kidney, coupled with the lack of strong $\mathrm{HH}$ response in epithelial cells in this model, suggest the existence of a separate cilia-mediated pathway in epithelial cells, disruption of which triggers a cascade of events leading to the formation of epithelial cysts and non-cell autonomous activation of $\mathrm{HH}$ signaling in interstitial cells. The relationship between this undefined ciliary pathway and the CDCA pathway, which also functions in epithelial cilia, is unknown. It will be interesting to investigate whether the CDCA pathway plays a role in non-cell autonomous activation of the $\mathrm{HH}$ pathway in cystic kidney models. Alternatively, 
Arl13b could function through an extraciliary pathway ${ }^{36}$. Whether this pathway is active in renal epithelial cells remains to be investigated.

Given the non-cell autonomous nature of $\mathrm{HH}$ activation in the Arll3bff; $;$ Ksp-Cre kidney, we sought to investigate the role of abnormal activation of $\mathrm{HH}$ signaling by using the small molecule GLI inhibitor GANT61 to reduce HH signaling globally. GANT61 treatment attenuated both renal fibrosis and cyst progression in $\mathrm{Arl13} \mathrm{b}^{\mathrm{fff}}$;Ksp-Cre mutants.

The impact on renal fibrosis is consistent with the role of $\mathrm{HH}$ in renal fibrosis triggered by kidney injury. Previous studies showed that $\mathrm{HH}$ signaling is activated after kidney injury and this activation is integral to repair ${ }^{10,12,33,37,38}$. Functionally, both global pharmacological inhibition of HH signaling and targeted genetic deletion of Shh in renal epithelial cells attenuated fibroblast activation after kidney injury ${ }^{12,33,37,38}$. GANT61 could similarly attenuate fibrosis in the $\mathrm{Arl} 13 b^{f f f} ; \mathrm{Ksp}$-Cre kidney through inhibiting fibroblast activation. The reduced level of $\alpha$ SMA and VIMENTIN in treated mutants is consistent with this model. Interestingly, GANT61 treatment also ameliorated cyst progression. Whether this effect is through reduced fibroblast activation or a more indirect mechanism such as macrophage recruitment, which has an established role in cyst progression, remains to be investigated ${ }^{39,40}$. Further, it will be vital for our understanding of PKD to test whether 
interstitial cells are also $\mathrm{HH}$ responsive cells in additional PKD models, including

Polycystin mutants and mutants affecting different aspects of cilia biogenesis and signaling.

\section{METHODS}

\section{Ethics statement}

Animal experiments in this study were carried out at Yale University School of Medicine in accordance with the Animal Use Protocols as approved by the Institutional Animal Care and Use Committee.

\section{Animals}

Arll3b fff Ksp-Cre mice have previously described ${ }^{29}$. Gli $1^{\text {lacZ }}$ mice (Jackson Laboratory,

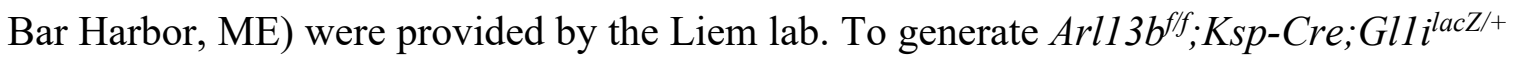

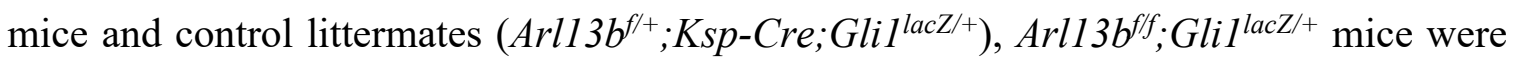
crossed to $\mathrm{Arl13} \mathrm{b}^{\mathrm{f} / \mathrm{+}}$; Ksp-Cre mice.

\section{GANT61 treatment}

GANT61 (ApexBio Technology, A1615, 25mg) was dissolved in ethanol (1.75 ml) and stored at $-80^{\circ} \mathrm{C}$. For mouse injection, the ethanol solution was further diluted in PBS $(3: 7)$ immediately before injection. GANT61 was intraperitoneally administered to male and female mice at a dose of $30 \mathrm{mg} / \mathrm{kg}$ body weight (BW)/day at both 10 and 11 days of age, 
and $50 \mathrm{mg} / \mathrm{kg} \mathrm{BW} /$ day at 12 to 20 days of age (Figure $4 \mathrm{~A}$ ). Control mice were injected with vehicle alone following the same schedule. Mice were sacrificed at P21.

\section{Cell culture}

mIMCD3 cells were cultured in Dulbecco's modified Eagle's medium/Nutrient Mixture F-12 (Invitrogen, 11330-032) supplemented with 10\% heat-inactivated fetal bovine serum (FBS, Invitrogen, 16410) and 1\% Antibiotic-Antimycotic solution (Gibco, 15240062). C3H/10T1/2, clone8 (10T1/2) cells were obtained from the American Type Culture Collection (ATCC, CCL-226) and was cultured in Basal Medium Eagle (BEM, Gibco, 21010046) supplemented with 10\% heat-inactivated FBS, 2mM L-glutamine (Gibco, 25030081) and 1\% Antibiotic-Antimycotic solution. Cells were maintained as a monolayer at sub-confluent densities in a $5 \% \mathrm{CO}_{2}$ humidified incubator at $37^{\circ} \mathrm{C}$. For immunofluorescence analysis, cells were seed into chambered cell culture slide (Fisher Scientific, 08-774-26). To induce primary cilium formation, cells were treated in lowserum $(0.5 \%$ FBS $)$ medium after reaching confluency and maintained for the duration indicated in each experiment. For Smoothened Agonist (SAG) treatment, SAG (Cayman Chemical, 11914) was dissolved in DMSO to make the stock solution (5mM). After one day of culturing in low serum medium $(0.5 \% \mathrm{FBS})$, cells were treated with varying concentrations of SAG in medium containing $0.5 \%$ FBS for 24 hours or 4 days as indicated in each experiment. The same amount of DMSO was used as vehicle control. 


\section{Western blot using cultured cells and mouse kidney tissues}

Cell pellets and kidney tissue samples were homogenized in whole cell extracts (WCE) buffer containing $20 \mathrm{mM}$ HEPES, pH 7.4, 0.2 M NaCl, 0.5\% Triton X-100, 5\% glycerol, 1 mM EDTA, 1 mM EGTA, 1 mM DTT, Phosphatase Inhibitor Cocktail (Thermo Fisher Scientific, 78440), and the protease inhibitor cocktail (Roche, 11697498001). Samples were lysed by trituration through a 25 -gauge needle 10 times on ice, rotated for 30 minutes at $4^{\circ} \mathrm{C}$, and centrifuged $\left(21000 \mathrm{~g}, 30\right.$ minutes, $\left.4^{\circ} \mathrm{C}\right)$ to obtain whole cell extracts. The protein concentration was determined by Bradford assay using protein assay dye (Bio-Rad). Lysates were boiled in 5x loading buffer (250 mM Tris-HCl, $\mathrm{pH} 6.8,10 \%$ SDS, 0.05\% bromophenol blue, 50\% glycerol, 25\% $\beta$-mercaptoethanol,) and then fractionated by SDS-PAGE. Western blot analysis was performed after electrophoretic separation of polypeptides by 4-15\% SDS-PAGE (Bio-Rad, 5000006) and transfer to ImmobilonP/PVDF membrane (Millipore, IPVH00010). Blots were probed overnight at $4^{\circ} \mathrm{C}$ with the following primary antibodies: rabbit anti-vimentin at 1:2000 (Proteintech Group Inc, 10366-1-AP), rabbit anti-collagen I at 1:2000 (Proteintech Group Inc, 14695-1-AP), mouse anti- $\alpha$ SMA at 1:2000 (Abcam, ab7817) and rabbit anti-GAPDH at 1:5000 (GeneTex, GTX100118). Following incubation with primary antibodies, the blots were washed and then probed with the respective HRP-conjugated secondary antibodies at 1:5000 (Cell Signaling Technology, 7076 and 7074). Immuno-bands were subsequently detected by the enhanced chemiluminescence reaction (Thermo Fisher Scientific, 34095). 


\section{Quantitative reserve transcription (RT) and real-time PCR}

Total RNA from cell pellets and kidney tissues were isolated using TRIzol reagent (Invitrogen, 1559602) according to manufacturer's instructions. cDNA was synthesized by Superscript II reverse transcriptase (Invitrogen, 18064071) with random hexamers. Primers

for real-time PCR assays are listed in Supplementary Table S1. cDNA levels were determined quantitatively by real-time PCR using the Bio-Rad iTaq Universal SYBR Green Supermix system (1725125) and normalized with Gapdh. All results represent the mean $\pm \mathrm{SD}$ of at least three independent experiments.

\section{Tissue preparation and histology}

Kidneys were fixed in 4\% paraformaldehyde (PFA) overnight at $4{ }^{\circ} \mathrm{C}$. For histological analysis, fixed tissues were embedded in paraffin wax, sectioned at $5 \mu \mathrm{m}$, and stained with hematoxylin and eosin (H\&E) or trichrome staining by the Research Histology Facility at Yale School of Medicine. For immunofluorescence studies, fixed tissues were embedded in OCT (Sakura Finetek, 4583) and cryosectioned into 5- $\mu \mathrm{m}$ sections by Microscopy Core of the Department of Cellular and Molecular Physiology at Yale School of Medicine.

\section{Immunofluorescence}

Cells seeded on slides were fixed in 4\% PFA for 5 min. Slides with OCT-embedded tissue sections and cultured cells were washed with PBS twice and subsequently permeabilized with $0.5 \%$ Triton X-100/0.1\% Tween-20/PBS at room temperature for 20 
min. Slides were then blocked in blocking buffer (R.T.U. Animal Free Block and Diluent, Vector Laboratories, SP-5035-100) and incubated overnight with the following primary antibodies: rabbit anti-laminin (1:200, NB300-144, Novus Biologicals), chicken anti-LacZ (1:3000, BGL-1010, Aves Labs), rabbit anti-vimentin (1:200), mouse anti- $\alpha$-SMA (1:200), rabbit anti-ARL13b (1:200, Proteintech Group, 17711-1-AP) and mouse anti-acetylated tubulin (1:5000, Sigma-Aldrich, clone 6-11B-1). Slides were washed three times in PBS and subsequently incubated for $1 \mathrm{~h}$ at room temperature in blocking buffer containing the secondary antibodies conjugated to Ig-Alexa Fluor 568, Ig-Alexa Fluor 488 or IgFluorescein. Slides were washed three times in 1XPBS and then mounted with Vectashield Vibrance mounting medium with DAPI (Vector Laboratories, H-1800).

\section{Cyst index analysis}

Cystic index was performed as previously described ${ }^{29,41}$. Cyst index was analyzed by Metamorph v.7.1 acquisition software (Universal Imaging) using H\&E-stained images in sagittal sections of kidneys. Cystic index was calculated by dividing the cyst-containing area by the total kidney area.

\section{Blood Urea Nitrogen (BUN) measurement}

Total blood was collected from mouse in a heparin-coated tube (BD Microtainer, BD Inc), and centrifuged (6000rpm, 5 minutes) to obtain plasma. Plasma BUN was measured 
using a Urea Nitrogen colorimetric detection kit (Invitrogen, EIABUN) following the manufacturer's instructions.

\section{Statistical analysis}

Data are presented as mean with error bars indicating the standard deviation (S.D.). Statistical significance was calculated by unpaired two-tailed t test and Pearson correlation analysis using GraphPad Prism 8.

\section{Disclosures:}

None.

\section{Funding:}

This work was supported by National Institute of Health grants R01DK113135 and R01HD093608 (to Dr. Sun).

\section{Acknowledgements:}

We thank the Liem lab for Gli ${ }^{l a c Z}$ mice; the members of Somlo laboratory and Sun

laboratory for helpful discussions; A. Cox for critical reading of the manuscript; S. 
bioRxiv preprint doi: https://doi.org/10.1101/2022.02.28.482403; this version posted March 1, 2022. The copyright holder for this preprint (which was not certified by peer review) is the author/funder. All rights reserved. No reuse allowed without permission.

Mentone in the Microscopy and Imaging Core of Cellular and Molecular Physiology

Department of Yale School of Medicine for histology assistance. 


\section{REFERENCES:}

1. Goetz SC, Anderson KV. The primary cilium: a signalling centre during vertebrate development. Nature reviews Genetics. May 2010;11(5):331-44. doi:10.1038/nrg2774

2. Wheway G, Nazlamova L, Hancock JT. Signaling through the Primary Cilium. Front Cell Dev Biol. 2018;6:8. doi:10.3389/fcell.2018.00008

3. Anvarian Z, Mykytyn K, Mukhopadhyay S, Pedersen LB, Christensen ST. Cellular signalling by primary cilia in development, organ function and disease. Nat Rev Nephrol. Apr 2019;15(4):199-219. doi:10.1038/s41581-019-0116-9

4. Haycraft CJ, Banizs B, Aydin-Son Y, Zhang Q, Michaud EJ, Yoder BK. Gli2 and Gli3 localize to cilia and require the intraflagellar transport protein polaris for processing and function. PLoS Genet. Oct 2005;1(4):e53.

5. Huangfu D, Liu A, Rakeman AS, Murcia NS, Niswander L, Anderson KV. Hedgehog signalling in the mouse requires intraflagellar transport proteins. Nature. Nov 6 2003;426(6962):83-7.

6. Rohatgi R, Milenkovic L, Scott MP. Patched1 regulates hedgehog signaling at the primary cilium. Science. Jul 20 2007;317(5836):372-6. doi:10.1126/science.1139740

7. Yu J, Carroll TJ, McMahon AP. Sonic hedgehog regulates proliferation and differentiation of mesenchymal cells in the mouse metanephric kidney. Development. Nov 2002;129(22):5301-12.

8. McMahon AP, Ingham PW, Tabin CJ. Developmental roles and clinical significance of hedgehog signaling. Curr Top Dev Biol. 2003;53:1-114. doi:10.1016/s00702153(03)53002-2

9. Lum L, Beachy PA. The Hedgehog response network: sensors, switches, and routers. Science. Jun 18 2004;304(5678):1755-9. doi:10.1126/science.1098020

10. Fabian SL, Penchev RR, St-Jacques B, et al. Hedgehog-Gli pathway activation during kidney fibrosis. The American journal of pathology. Apr 2012;180(4):1441-53. doi:10.1016/j.ajpath.2011.12.039

11. Horn A, Palumbo K, Cordazzo C, et al. Hedgehog signaling controls fibroblast activation and tissue fibrosis in systemic sclerosis. Arthritis Rheum. Aug 2012;64(8):2724-33. doi:10.1002/art.34444 
12. Zhou D, Li Y, Zhou L, et al. Sonic hedgehog is a novel tubule-derived growth factor for interstitial fibroblasts after kidney injury. Journal of the American Society of Nephrology : JASN. Oct 2014;25(10):2187-200. doi:10.1681/ASN.2013080893

13. Hu B, Liu J, Wu Z, et al. Reemergence of hedgehog mediates epithelial-mesenchymal crosstalk in pulmonary fibrosis. Am J Respir Cell Mol Biol. Apr 2015;52(4):418-28. doi: $10.1165 / \mathrm{rcmb} .2014-0108 \mathrm{OC}$

14. Jung IH, Jung DE, Park YN, Song SY, Park SW. Aberrant Hedgehog ligands induce progressive pancreatic fibrosis by paracrine activation of myofibroblasts and ductular cells in transgenic zebrafish. PLoS One. 2011;6(12):e27941. doi:10.1371/journal.pone.0027941

15. Waters AM, Beales PL. Ciliopathies: an expanding disease spectrum. Pediatr Nephrol. Jul 2011;26(7):1039-56. doi:10.1007/s00467-010-1731-7

16. Novarino G, Akizu N, Gleeson JG. Modeling human disease in humans: the ciliopathies. Cell. Sep 30 2011;147(1):70-9. doi:10.1016/j.cell.2011.09.014

17. Hildebrandt F, Benzing T, Katsanis N. Ciliopathies. N Engl J Med. Apr 21 2011;364(16):1533-43. doi:10.1056/NEJMra1010172

18. Chang CF, Chang YT, Millington G, Brugmann SA. Craniofacial Ciliopathies Reveal Specific Requirements for GLI Proteins during Development of the Facial Midline. PLoS Genet. Nov 2016;12(11):e1006351. doi:10.1371/journal.pgen.1006351

19. Liu A, Wang B, Niswander LA. Mouse intraflagellar transport proteins regulate both the activator and repressor functions of Gli transcription factors. Development. Jul 2005;132(13):3103-11. doi:10.1242/dev.01894

20. Bergmann C, Guay-Woodford LM, Harris PC, Horie S, Peters DJM, Torres VE. Polycystic kidney disease. Nat Rev Dis Primers. Dec 6 2018;4(1):50. doi:10.1038/s41572-018-0047-y

21. Barr MM, Sternberg PW. A polycystic kidney-disease gene homologue required for male mating behaviour in C. elegans. Nature. Sep 23 1999;401(6751):386-9. doi:10.1038/43913

22. Cai Y, Fedeles SV, Dong K, et al. Altered trafficking and stability of polycystins underlie polycystic kidney disease. J Clin Invest. Dec 2014;124(12):5129-44. doi:10.1172/JCI67273 
23. Pazour GJ, San Agustin JT, Follit JA, Rosenbaum JL, Witman GB. Polycystin-2 localizes to kidney cilia and the ciliary level is elevated in orpk mice with polycystic kidney disease. Current biology : CB. Jun 4 2002;12(11):R378-80.

24. Yoder BK, Hou X, Guay-Woodford LM. The polycystic kidney disease proteins, polycystin-1, polycystin-2, polaris, and cystin, are co-localized in renal cilia. Journal of the American Society of Nephrology : JASN. Oct 2002;13(10):2508-16.

25. Yoshiba S, Shiratori H, Kuo IY, et al. Cilia at the node of mouse embryos sense fluid flow for left-right determination via Pkd2. Science. Oct 12 2012;338(6104):226-31. doi: $10.1126 /$ science. 1222538

26. Ma M, Tian X, Igarashi P, Pazour GJ, Somlo S. Loss of cilia suppresses cyst growth in genetic models of autosomal dominant polycystic kidney disease. Nat Genet. Sep 2013;45(9):1004-12. doi:10.1038/ng.2715

27. Tran PV, Talbott GC, Turbe-Doan A, et al. Downregulating Hedgehog Signaling Reduces Renal Cystogenic Potential of Mouse Models. Journal of the American Society of Nephrology : JASN. Apr 3 2014;doi:10.1681/ASN.2013070735

28. Silva LM, Jacobs DT, Allard BA, et al. Inhibition of Hedgehog signaling suppresses proliferation and microcyst formation of human Autosomal Dominant Polycystic Kidney Disease cells. Sci Rep. Mar 21 2018;8(1):4985. doi:10.1038/s41598-018$23341-2$

29. Li Y, Tian X, Ma M, et al. Deletion of ADP Ribosylation Factor-Like GTPase 13B Leads to Kidney Cysts. Journal of the American Society of Nephrology : JASN. May 6 2016;doi:10.1681/ASN.2015091004

30. Jonassen JA, SanAgustin J, Baker SP, Pazour GJ. Disruption of IFT complex A causes cystic kidneys without mitotic spindle misorientation. Journal of the American Society of Nephrology : JASN. Apr 2012;23(4):641-51. doi:10.1681/ASN.2011080829

31. Tran PV, Haycraft CJ, Besschetnova TY, et al. THM1 negatively modulates mouse sonic hedgehog signal transduction and affects retrograde intraflagellar transport in cilia. Nat Genet. Apr 2008;40(4):403-410. doi:10.1038/ng.105

32. Ma M, Legue E, Tian X, Somlo S, Liem KF, Jr. Cell-Autonomous Hedgehog Signaling Is Not Required for Cyst Formation in Autosomal Dominant Polycystic Kidney Disease. Journal of the American Society of Nephrology : JASN. Nov 2019;30(11):2103-2111. doi:10.1681/ASN.2018121274 
33. Ding H, Zhou D, Hao S, et al. Sonic hedgehog signaling mediates epithelialmesenchymal communication and promotes renal fibrosis. Journal of the American Society of Nephrology : JASN. May 2012;23(5):801-13. doi:10.1681/ASN.2011060614

34. Bai CB, Auerbach W, Lee JS, Stephen D, Joyner AL. Gli2, but not Gli1, is required for initial Shh signaling and ectopic activation of the Shh pathway. Development. Oct 2002;129(20):4753-61.

35. Lauth M, Bergstrom A, Shimokawa T, Toftgard R. Inhibition of GLI-mediated transcription and tumor cell growth by small-molecule antagonists. Proc Natl Acad Sci U S A. May 15 2007;104(20):8455-60. doi:10.1073/pnas.0609699104

36. Gigante ED, Taylor MR, Ivanova AA, Kahn RA, Caspary T. ARL13B regulates Sonic hedgehog signaling from outside primary cilia. Elife. Mar 4 2020;9doi:10.7554/eLife.50434

37. Kramann R, Fleig SV, Schneider RK, et al. Pharmacological GLI2 inhibition prevents myofibroblast cell-cycle progression and reduces kidney fibrosis. J Clin Invest. Aug 3 2015;125(8):2935-51. doi:10.1172/JCI74929

38. Zhou D, Fu H, Liu S, et al. Early activation of fibroblasts is required for kidney repair and regeneration after injury. FASEB J. Nov 2019;33(11):12576-12587. doi:10.1096/fj.201900651RR

39. Karihaloo A, Koraishy F, Huen SC, et al. Macrophages promote cyst growth in polycystic kidney disease. Journal of the American Society of Nephrology : JASN. Oct 2011;22(10):1809-14. doi:10.1681/ASN.2011010084

40. Swenson-Fields KI, Vivian CJ, Salah SM, et al. Macrophages promote polycystic kidney disease progression. Kidney Int. May 2013;83(5):855-64. doi:10.1038/ki.2012.446

41. Shibazaki S, Yu Z, Nishio S, et al. Cyst formation and activation of the extracellular regulated kinase pathway after kidney specific inactivation of Pkd1. Hum Mol Genet. Jun 1 2008;17(11):1505-16. doi:10.1093/hmg/ddn039 

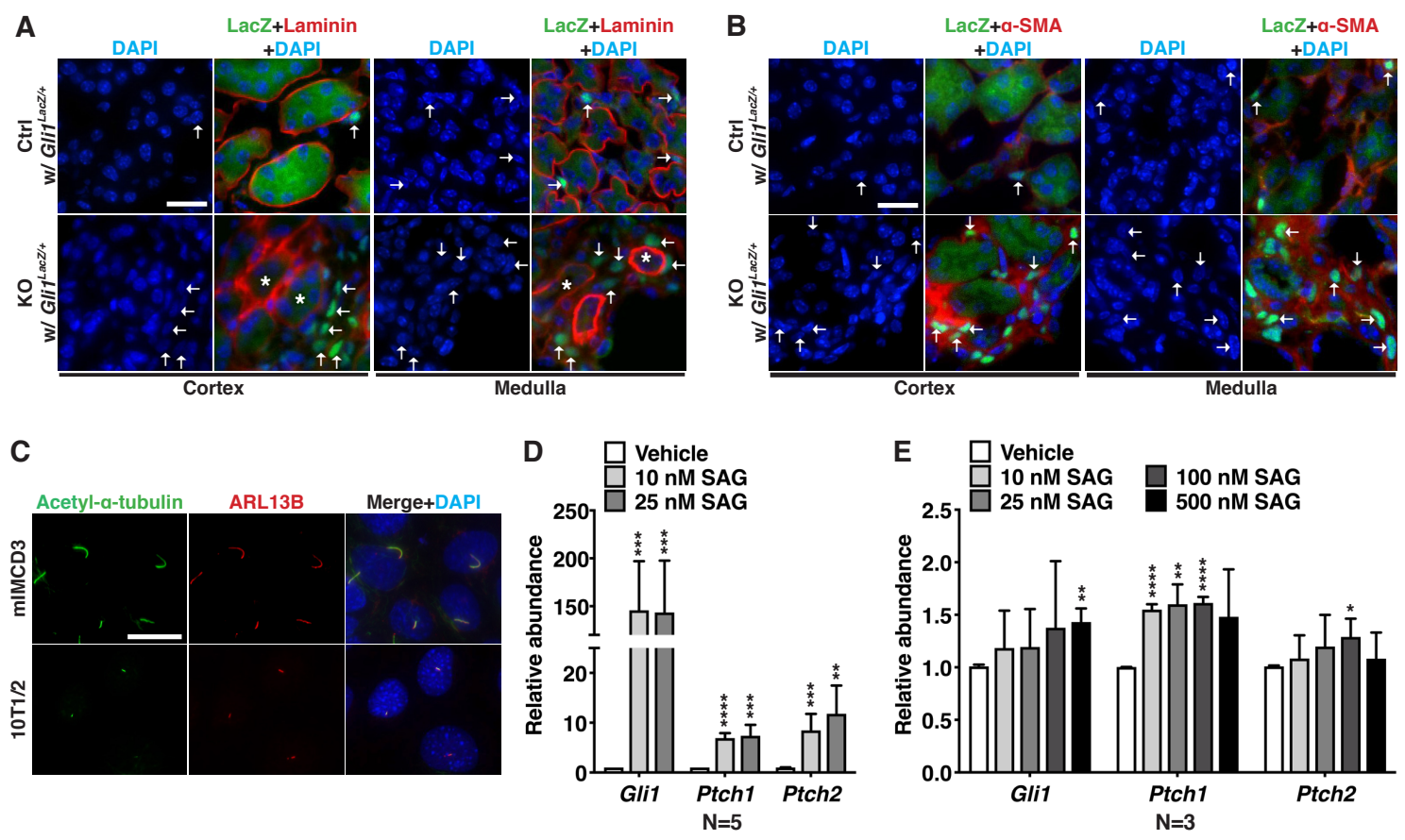

Figure 1. Epithelial-specific inactivation of $A r l 13 b$ results in non-cell autonomous activation of $\mathrm{HH}$ signaling in stromal cells. (A, B) Immunofluorescent staining of kidney

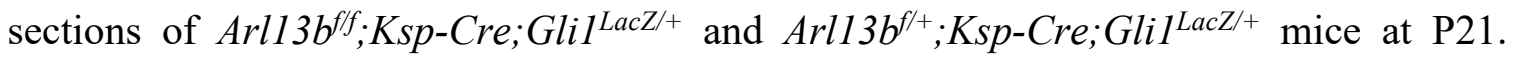
Cells with active HH signaling are indicated by nLacZ signal (green, detected by anti-LacZ) Arrows point to representative $\mathrm{nLacZ}$ positive nuclei. Stars indicate $\mathrm{nLacZ}$ negative epithelial region encircled by Laminin. DAPI = nuclear counterstain (blue). Scale bars: 20 $\mu \mathrm{m}$. In (A), anti-Laminin (red) outlines the basal border of epithelial tubules. In (B), anti$\alpha$-SMA (red) labels activated myofibroblasts. (C) Cilia in mIMCD3 and 10T1/2 cells detected by anti-acetylated tubulin (green) and anti-ARL13B (red). Nuclei stained with DAPI (blue). Scale bar: $10 \mu \mathrm{m}$. (D, E) QRT-PCR analysis of the expression level of HH target genes in 10T1/2 (D) and mIMCD3 (E) cells in response to SAG treatment. Unit 1 is defined by the expression level in vehicle treated controls. Results represent mean $\pm \mathrm{SD}$ of at least three independent experiments. Ctrl: $A r l 13 b^{f /+} ; K s p-C r e$ mice; KO: Arll3b ${ }^{f f f} ; \mathrm{Ksp}$ Cre mice; ${ }^{*} \mathrm{P}<0.05 ; * * \mathrm{P}<0.01 ; * * * \mathrm{P}<0.001 ; * * * * \mathrm{P}<0.0001$. All scale bars: $20 \mu \mathrm{m}$. 
A

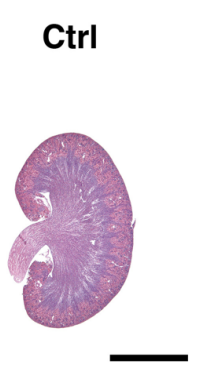

D

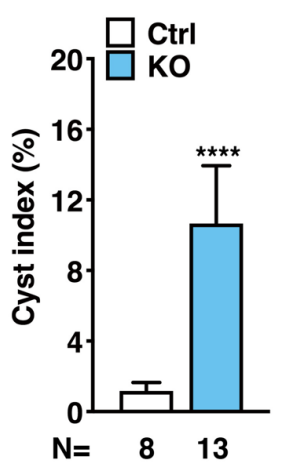

B

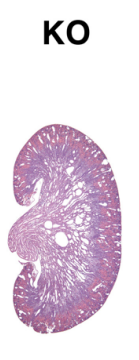

$\mathbf{E}$

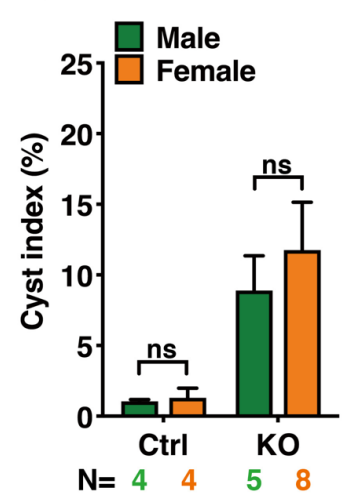

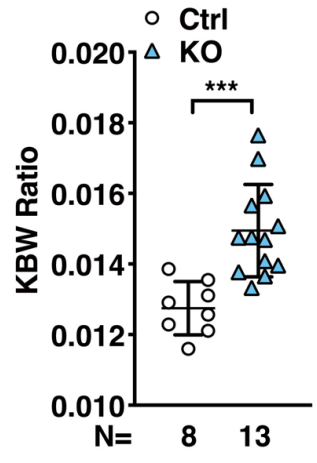

$\mathbf{F}$

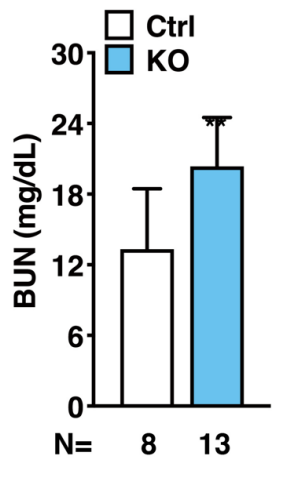

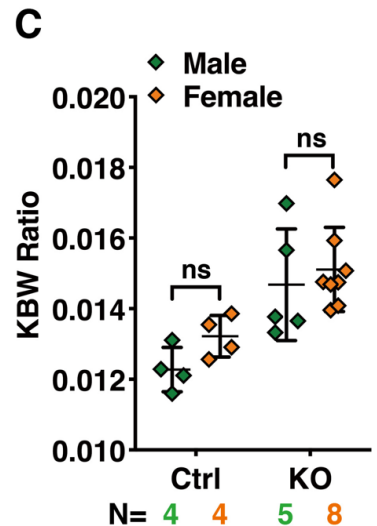

G

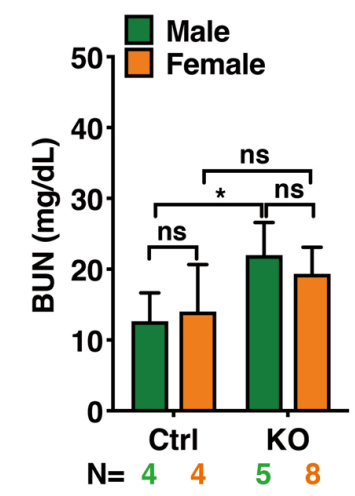

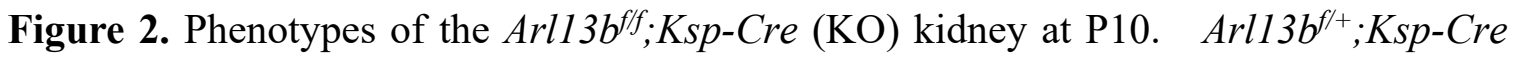
mice are used as control (Ctrl). Scale bar: $2 \mathrm{~mm}$. (A) Hematoxylin and eosin-stained kidney sections. (B, C) KBW in $\mathrm{KO}$ and $\mathrm{Ctrl}$ mice. (D, E) Cystic index in $\mathrm{KO}$ and $\mathrm{Ctrl}$ mice). (F, G) BUN level in KO and Ctrl mice. ${ }^{*} \mathrm{P}<0.05 ; * * \mathrm{P}<0 / 01$; $* * * * \mathrm{P}<0.0001$; ns, $\mathrm{P}>0.05$. 
A

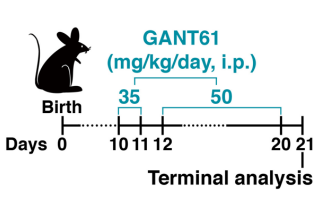

D

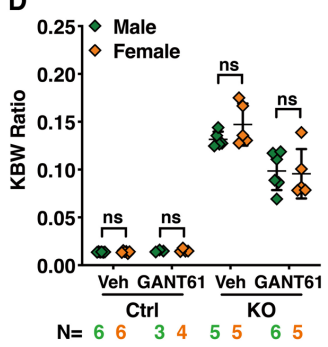

G

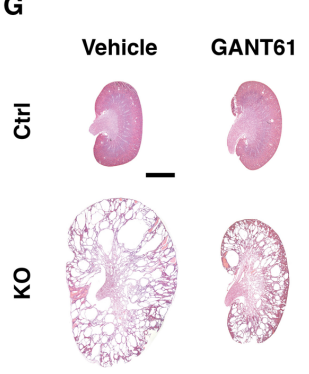

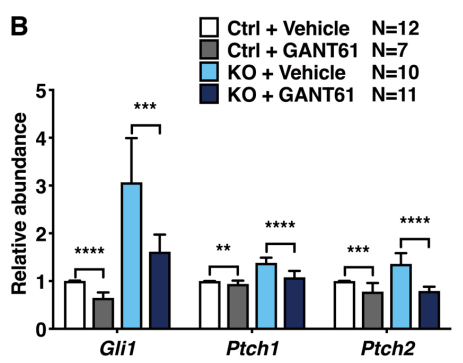

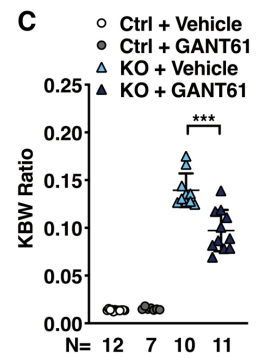

E
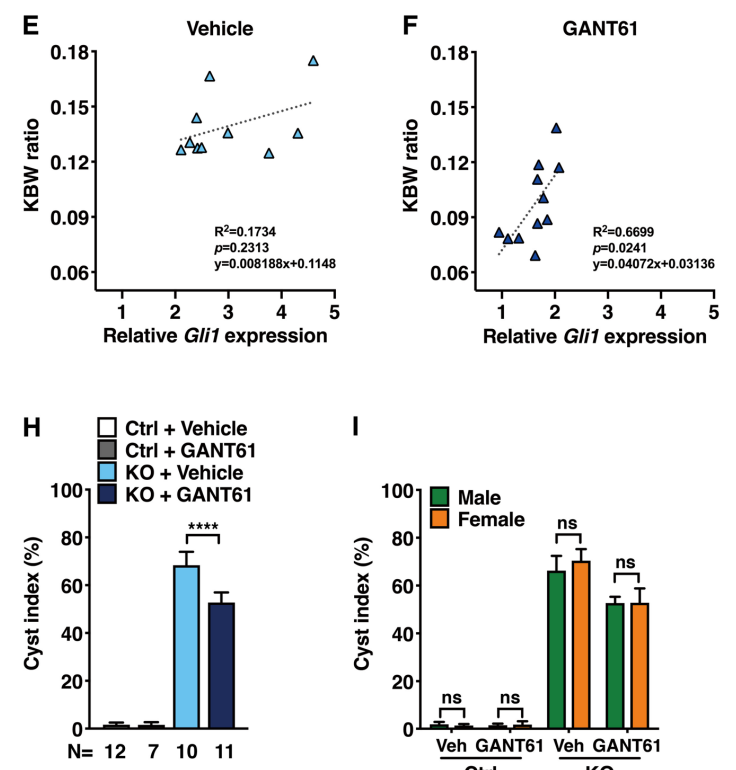

I

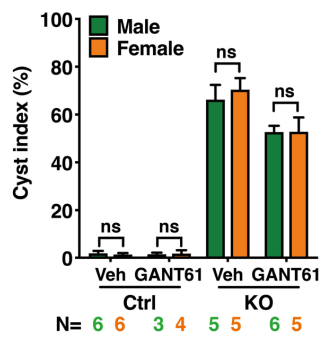

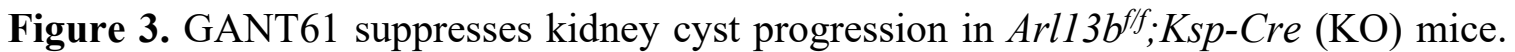
(A) Schematic diagram of the treatment schedule. P10 and P11 mice were subjected to daily i.p. injection of $35 \mathrm{mg} / \mathrm{kg} /$ day GANT61, followed by daily i.p. injection of 50 $\mathrm{mg} / \mathrm{kg} /$ day GANT61 from P12 to P20. (B) GANT61 treatment reduced the expression level of HH target genes Gli1, Ptch1 and Ptch 2 in whole kidney lysates of KO and $\mathrm{Arll} 3 \mathrm{~b}^{f /{ }^{+}} ; \mathrm{Ksp}$ Cre (Ctrl) mice, assayed by qRT-PCR. Gapdh was used for normalization. Unit 1 is defined by the expression level in vehicle treated animals. Results represent mean $\pm \mathrm{SD}$ of at least three independent experiments. (C, D) KWB ratio in vehicle (Veh) and GANT61 treated mutant KO and $\mathrm{Arll} 3 \mathrm{~b}^{f /+} ; \mathrm{Ksp}-\mathrm{Cre}(\mathrm{Ctrl})$ mice. (E, F) Pearson correlation between the level of whole kidney Glil expression and KWB in vehicle (E) and GANT61 (F) treated Arl13 $b^{f f} ; K s p$-Cre mice. (F) Hematoxylin and eosin-stained kidney sections from GANT61 and vehicle $\mathrm{Arll} 3 \mathrm{~b}^{f /+} ; \mathrm{Ksp}-\mathrm{Cre}(\mathrm{Ctrl})$ and $\mathrm{KO}$ mice. Scale bar: $2 \mathrm{~mm}$. (G, I) Cystic index of GANT61 and vehicle (Veh) treated $A r l 13 b^{f /+} ; K s p-C r e(C t r l)$ and KO kidneys. ${ }^{* *} \mathrm{P}<0.01$; $* * * \mathrm{P}<0.001 ; * * * * \mathrm{P}<0.0001 ; \mathrm{ns}, \mathrm{P}>0.05$. 
A

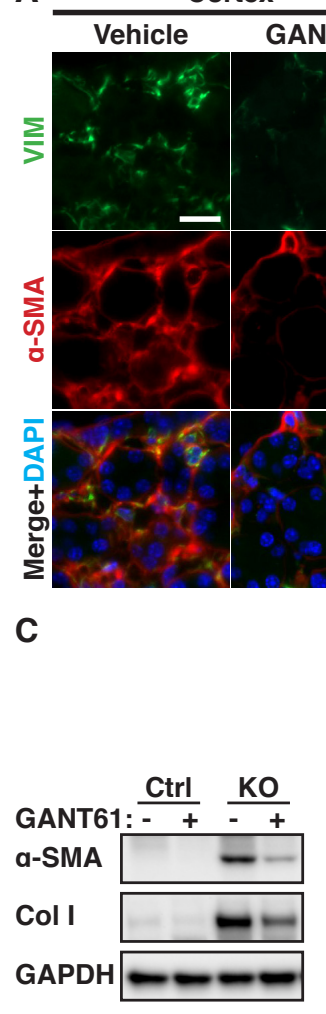

Medulla

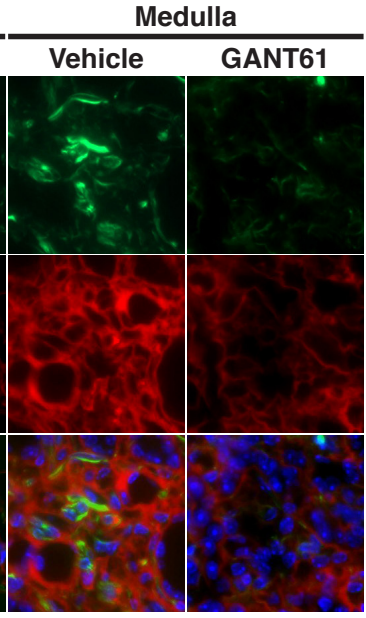

D

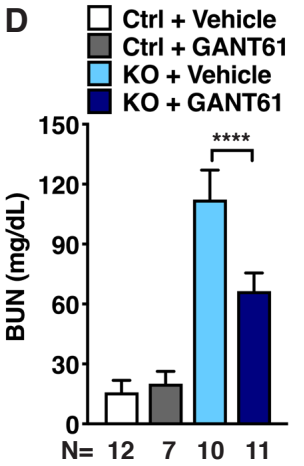

B
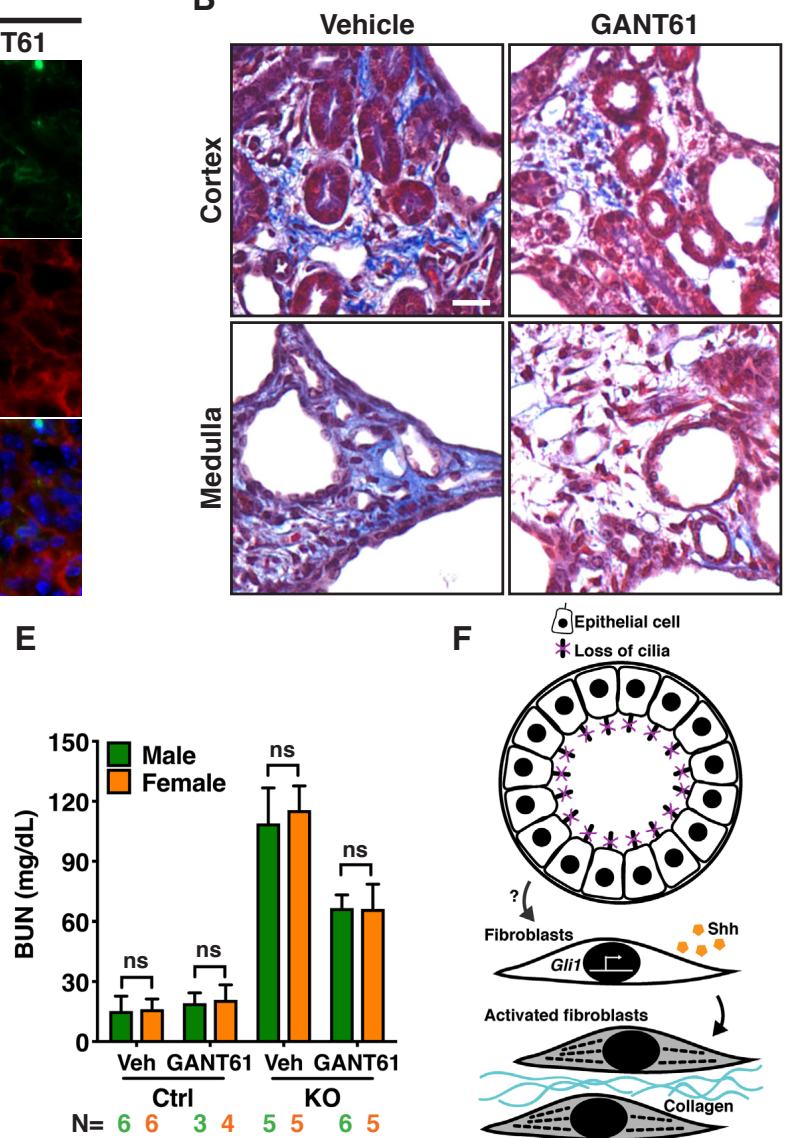

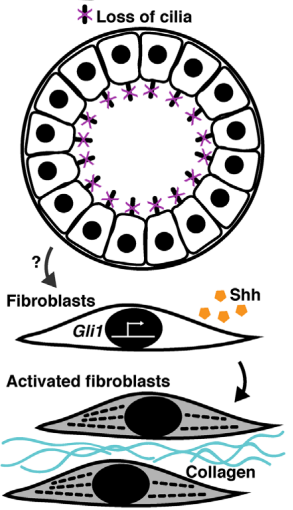

Figure 4. GANT61 suppresses renal fibrosis and improves kidney function in Arll3b fff; Ksp-Cre (KO) mice. (A) Immunofluorescent staining of kidney sections of vehicle- and GANT61-treated KO mice. Anti-VIM in green, anti- $\alpha$ SMA in red. DAPI, blue. Scale bar: $20 \mu \mathrm{m}$. (B) Trichrome-stained kidney sections of vehicle- and GANT61-treated KO mice. Blue color indicates collagen deposition. Scale bar: $20 \mu \mathrm{m}$. (C) Western blot showing the protein level of $\alpha \mathrm{SMA}$ and collagen I (Col I) in whole kidney lysates of $\mathrm{KO}$ mice treated with GANT61 (+) or vehicle (-). GAPDH is used as a loading control. (D, E) BUN level in GANT61 or vehicle treated $\mathrm{KO}$ and $\mathrm{Arl13} \mathrm{b}^{f /+} ; \mathrm{Ksp}-\mathrm{Cre}(\mathrm{Ctrl})$ mice. Male and female combined is shown in D and separately in E. Results represent the mean $\pm \mathrm{SD}$ of at least three independent experiments. $* * * * \mathrm{P}<0.0001$; ns, $\mathrm{P}>0.05$. (F) A model showing the non-cell autonomous activation of $\mathrm{HH}$ signaling in stromal cells. 
Supplementary Table S1: Primers used in qRT-PCR:

$\begin{array}{ll}\text { Gli1-F } & \text { CCCGGGTTATGGAGCAGCCAGA } \\ \text { Gli1-R } & \text { CTGGCATCAGAAAGGGGCGAGA } \\ \text { Ptch1-F } & \text { TGGCCGCATTGATCCCTATC } \\ \text { Ptch1-R } & \text { ACACAGGGGCTTGTGAAACA } \\ \text { Ptch2-F } & \text { TGGTAATCCTCGTGGCCTCT } \\ \text { Ptch2-R } & \text { AACCAGCAAGCATGAGCAGA } \\ \text { Ihh-F } & \text { CGGCTTCGACTGGGTGTATT } \\ \text { Ihh-R } & \text { CATCACTGAAGGTGGGGGTC } \\ \text { Dhh-F } & \text { CGTACCCAACTACAACCCCG } \\ \text { Dhh-R } & \text { GTGGAGTGAATCCTGTGCGT } \\ \text { Shh-F } & \text { AGACCGGCTGATGACTCAGAG } \\ \text { Shh-R } & \text { GCTCGACCCTCATAGTGTAGAGAC } \\ \text { GAPDH-F } & \text { GCAATGCATCCTGCACCACCA } \\ \text { GAPDH-R } & \text { TTCCAGAGGGGCCATCCACA }\end{array}$

